\title{
Development of Digital FLL System for SQUID Using Double Counter Method
}

\author{
D. Oyama ${ }^{1}$, K. Kobayashi ${ }^{1}$, M. Yoshizawa ${ }^{1}$, and Y. Uchikawa ${ }^{2}$ \\ ${ }^{1}$ Faculty of Engineering, Iwate University, Iwate 020-8551, Japan \\ ${ }^{2}$ College of Science and Engineering, Tokyo Denki University, Saitama 350-0394, Japan
}

\begin{abstract}
We have developed a digitally controlled flux-locked loop (FLL) system using a double-counter method to achieve both high performance and low cost. This system is composed of generally used electric devices. The system slew rate is about $1.3 \mathrm{k} \Phi_{0} / \mathrm{s}$, where $\Phi_{0}$ is the flux quantum. The measurable flux amplitude and dynamic range are $218 \Phi_{0}$ and $141 \mathrm{~dB}$ at $1 \mathrm{~Hz}$ and $22 \Phi_{0}$ and $121 \mathrm{~dB}$ at $10 \mathrm{~Hz}$, respectively. A white noise level measured in the magnetically shielded room (MSR) is $36.1 \mathrm{fT} / \mathrm{Hz}^{1 / 2}$. Magnetic measurements with a first-order gradiometer were done outside the MSR, and the developed system can detect a magnetocardiogram in an unshielded condition.
\end{abstract}

Index Terms-Double-counter method, flux-locked loop (FLL), magnetically unshielded, magnetocardiogram, SQUID.

\section{INTRODUCTION}

B IOMAGNETIC field measurements in an unshielded environment require both a large dynamic range and a high slew rate. Environmental interferences (e.g., power lines) commonly exhibit much larger amplitudes of about $100 \Phi_{0}$, where $\Phi_{0}$ is the flux quantum, even if the magnetometer is operated unshielded in a magnetically "quiet" environment [1].

In recent years, it has been demonstrated by several groups that digital SQUID electronics with a digitally controlled fluxlocked loop (FLL) system achieve such performances [1]-[3]. We call a digitally controlled flux-locked loop system "D-FLL."

However, these systems are mainly used for a magnetoencephalogram (MEG) measurement system [4]. Not only high performance but also low cost is necessary to expand biomagnetic measurement, especially magnetocardiogram (MCG) measurement.

In this paper, we propose a double-counter method to make a D-FLL system have high performance and low cost. The doublecounter D-FLL system is composed by a generally used onechip microcontroller and some inexpensive devices. We carried out characteristic evaluation and demonstrations of MCG measurement outside a magnetically shielded room (MSR).

\section{PRINCIPLE}

The flux-quanta counting method is used for increasing the dynamic range [1]. This method is based on the periodicity of the SQUID voltage-flux $(V-\Phi)$ characteristic. The integrator of an FLL is reset at every time when the feedback flux exceeds $\pm 1 \Phi_{0}$ and the corresponding $\Phi_{0}$ steps are counted.

Usually, the original input flux is reconstructed on a digital signal processor on FLL electronics. The dynamic range depends on the architecture of the digital signal processor, typically 32 bits. Furthermore, high-speed signal processing makes a digital feedback loop delay shorter. A high-performance digital signal processor is required to increase the dynamic range

Digital Object Identifier 10.1109/TMAG.2006.879162 and slew rate. So, it has been difficult to make a high-performance system by a generally used one-chip microcontroller.

Fig. 1 shows a block diagram of the double-counter D-FLL system. The double-counter method has two counters on a onechip microcontroller and a host computer. An integrator unit has a digital integrator that calculates feedback flux data. If feedback flux exceeds $\pm 0.5 \Phi_{0}$, integrator unit subtracts $1 \Phi_{0}$ from digital integrator data or adds to it, instead of "FLL reset." The feedback loop processing works more quickly than data transmission from the one-chip microcontroller into a host computer. The integrator data and Coutner 1 data are sent according to time interval generated by a timer. The Counter1 is reset every time when this transmission is done and then counts $\Phi_{0}$ steps during this interval. The Counter 2 integrates the data transmitted from Counter1. The host computer reconstructs the measured flux data from Counter 2 and integrator data.

Because a host computer reconstructs input flux, a workload of one-chip microcontroller is decreased. Then the feedback loop becomes quicker than without a double-counter method. The high-performance D-FLL system using a one-chip microcontroller is achieved by a double-counter method.

\section{EXPERIMENTAL PERFORMANCE}

Our latest D-FLL system is composed by a generally used 8-bit one-chip microcontroller, a 12-bit A/D converter, two 16-bit D/A converters, and some inexpensive and easily obtained devices. The bias current of a SQUID is a de signal generated by the microcontroller. The measured magnetic flux data are recorded at $1 \mathrm{kHz}$ through RS232. A time delay of the digital feedback loop is $8.4 \mu \mathrm{s}$.

The low- $T_{c}$ dc SQUID is used for the characteristic evaluations of the D-FLL system. It has a first-order gradiometer as a pickup coil, whose diameter is $17.8 \mathrm{~mm}$ and a baseline is 50 $\mathrm{mm}$. A sensitivity $B / \Phi=0.6 \mathrm{nT} / \Phi_{0}$, where $B$ is the flux density in the pickup coil and a flux resolution of the system is 11.35 fT/digit (19 $\mu \Phi_{0} /$ digit).

Fig. 2 shows an active area of the D-FLL system. It means the system can measure magnetic signal just in this area. The right vertical axis is shown as a dynamic range when a 16-bit D/A converter is used for about $1 \Phi_{0}$ feedback flux. The measured 


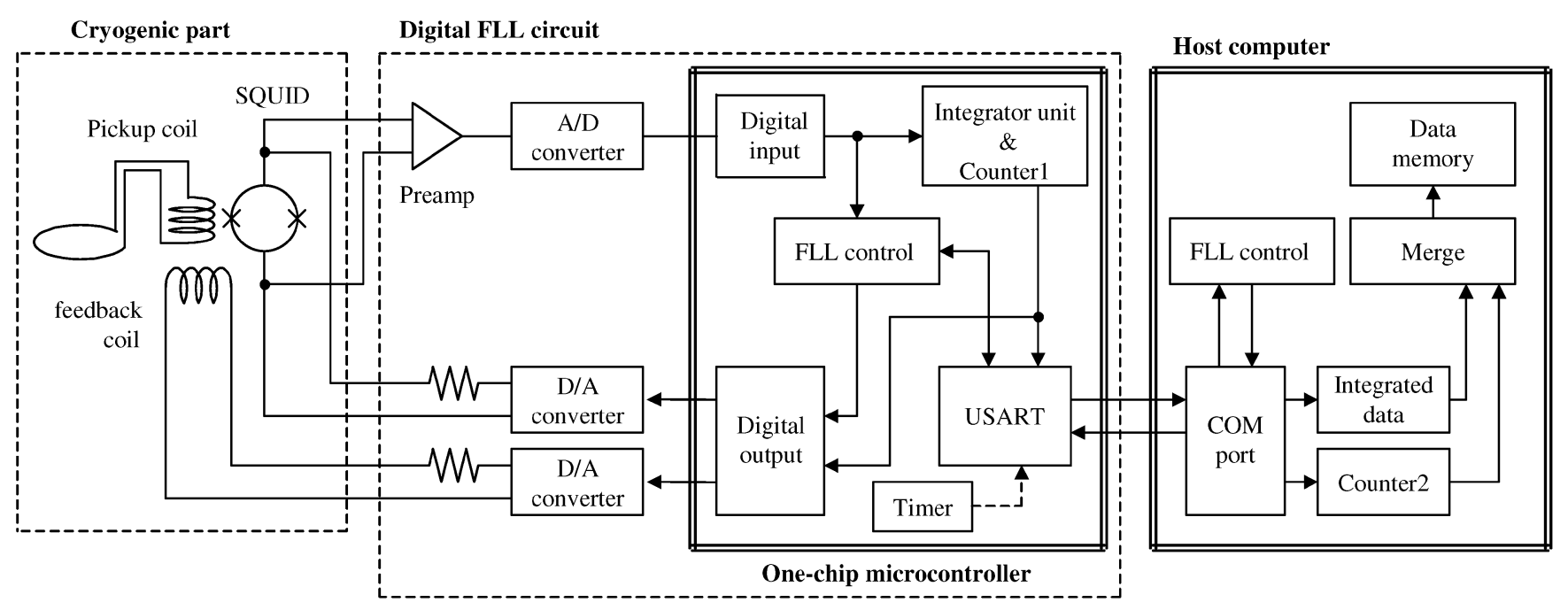

Fig. 1. Block diagram of the double-counter D-FLL system.

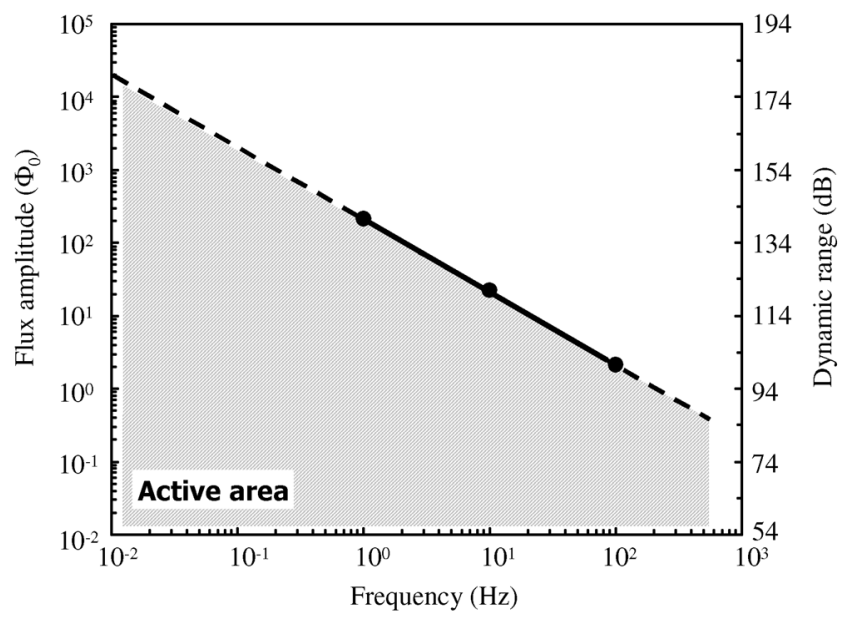

Fig. 2. Active area of the D-FLL system. It means the system can measure magnetic signal just in this area. The right vertical axis is shown as a dynamic range when a 16-bit D/A converter is used for $1 \Phi_{0}$ feedback flux.

dynamic range is $218 \Phi_{0}(141 \mathrm{~dB})$ at $1 \mathrm{~Hz}, 22 \Phi_{0}(121 \mathrm{~dB})$ at 10 $\mathrm{Hz}$, and $2.1 \Phi_{0}(100 \mathrm{~dB})$ at $100 \mathrm{~Hz}$. Also, a measured slew rate is $1.3 \mathrm{k} \Phi_{0} / \mathrm{s}$. The D-FLL system can operate stably if power line noise that is usually a main problem for unshielded operation is less than $4.1 \Phi_{0}$ amplitude at $50 \mathrm{~Hz}$.

Fig. 3 shows measured D-FLL output noise spectra inside and outside MSR. The lower line is measured in the MSR, and the upper is measured in the unshielded environment. A white noise level was $36.1 \mathrm{fT} / \mathrm{Hz}^{1 / 2}$, and a $1 / f$ corner frequency was $2 \mathrm{~Hz}$ in an MSR. Noise spectrum measured outside an MSR contained some uncertain noises beside power line noise that exhibited $465 \mathrm{pT} / \mathrm{Hz}^{1 / 2}\left(0.78 \Phi_{0} / \mathrm{Hz}^{1 / 2}\right)$ at $50 \mathrm{~Hz}$. These measurements are done at the Advanced Science and Technology Institute of Iwate.

\section{UNSHIELDED OPERATION}

Figs. 4 and 5 show experimental results obtained with doublecounter method D-FLL system. SQUID connected to a firstorder gradiometer, which was described above, was cooled inside an MSR.

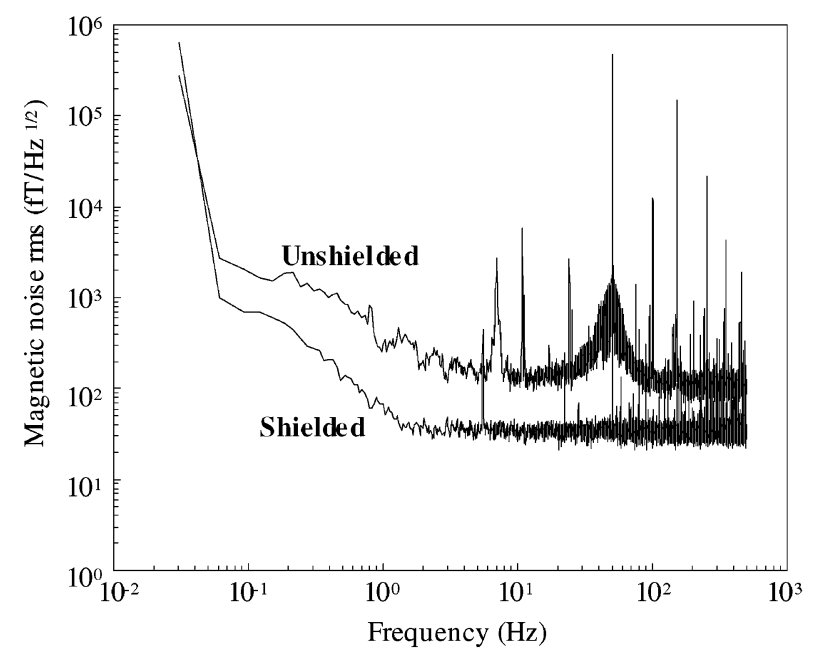

Fig. 3. Magnetic noise spectra measured in an MSR. The lower line is measured in an MSR, and the upper line is measured in the unshielded environment.

Fig. 4 shows a magnetic field of D-FLL operation both inside and outside an MSR. The D-FLL operation is started in the MSR, then taken outside, and taken back into the MSR.

This demonstration was continuously done two times. Fig. 4(a) shows a waveform of the whole time, Fig. 4(b) is a close-up of this waveform while the SQUID sensor has stayed in the MSR, and Fig. 4(c) is that of outside the MSR. The system remained locked all the time, and this waveform shows reproducibility. Four peaks in Fig. 4(a) are detected near a wall of the MSR.

Fig. 5 shows an MCG measurement outside an MSR. This waveform is obtained by applying a software band-pass filter (1 to $40 \mathrm{~Hz}$ ) and 199 times averaging. QRS complex is shown, and this figure proves that the D-FLL system can detect MCG waveform outside an MSR.

However, the D-FLL system needs to be improved in the area of dynamic and noise performance for practical MCG application. High slew rate will be achieved by well designing the components of digitally feedback loop: preamp, time delay of the feedback loop, and resolution of A/D converter. Furthermore, 

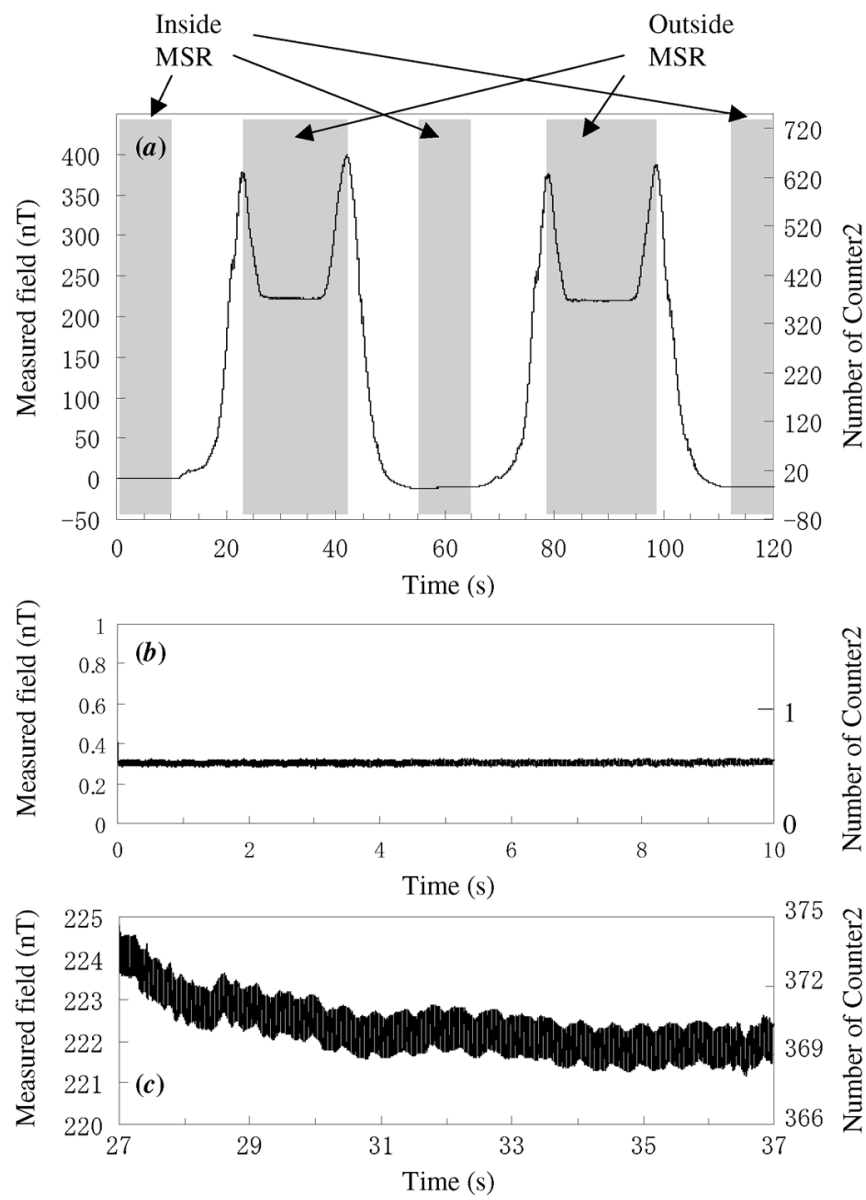

Fig. 4. Magnetic field of unshielded operation. Magnetic field measured (a) inside and outside an MSR, $(b)$ in the MSR, and $(c)$ outside the MSR. Each right vertical axis shows a number of Counter2. Low- $T_{c}$ SQUID sensor went out of the MSR and back into the MSR twice.

noise performance will be improved by a statistical signal processing using multichannel signals, like independent component analysis (ICA)[5], singular value decomposition (SVD)[6], and so on. This double-counter D-FLL system is well suited for a multichannel system.

\section{CONCLUSION}

We proposed a double-counter method to achieve a high performance and low cost D-FLL system. It can measure MCG

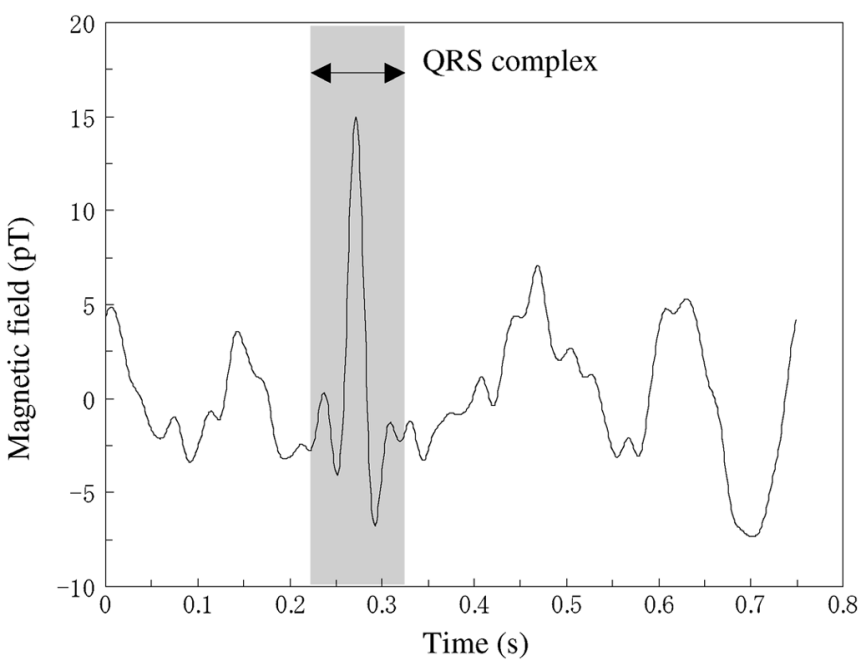

Fig. 5. MCG waveform measured in unshielded environment. The waveform is obtained by applying a software band-pass filter $(1 \mathrm{~Hz}$ to $40 \mathrm{~Hz})$ and 199 times averaging.

waveform outside an MSR. However, it was still insufficient to be used for an MCG diagnosis. It is necessary to improve the dynamic range and noise performance.

\section{REFERENCES}

[1] D. Drung, "High- $T_{c}$ and low- $T_{c}$ dc SQUID electronics," Supercond. Sci. Technol, vol. 16, pp. 1320-1336, 2003.

[2] C. Ludwig, C. Kessler, A. J. Steinfort, and W. Ludwig, "Versatile high performance digital SQUID electronics," IEEE Trans. Appl. Supercond., pt. 1, vol. 11, no. 1, pp. 1122-1125, Mar. 2001.

[3] T. Schurig, D. Drung, S. Bechstein, J. Beyer, and F. Ludwig, "High- $T_{c}$ superconductor dc SQUID's for unshielded operation and their applications," Physica C, vol. 378-381, pp. 1378-1384, 2002.

[4] J. Vrba and S. E. Robinson, "Signal processing in magnetoencephalography," Methods, vol. 25, pp. 249-271, 2001.

[5] K. Kobayashi, Y. Uchikawa, T. Simizu, K. Nakai, and M. Yoshizawa, "The rejection of magnetic noise from the wire using independent component analysis for magnetocardiogram," IEEE Trans. Magn., vol. 41, no. 10 , pp. 4152-4154, Oct. 2005.

[6] K. Kobayashi and Y. Uchikawa, "Estimation of multiple sources using spatio-temporal data on a three-dimensional measurement of MEG," IEEE Trans. Magn., vol. 37, no. 4, pp. 2915-2917, Jul. 2001.

Manuscript received March 13, 2006 (e-mail: t3704008@iwate-u.ac.jp). 